\title{
LETTER \\ Channel Estimation for OFDM-Based WLANs in the Presence of Wiener Phase Noise and Residual Frequency Offset
}

\author{
Yong-Hwa KIM ${ }^{\dagger}$, Jong-Ho LEE ${ }^{\dagger}$, Nonmembers, and Seong-Cheol KIM ${ }^{\dagger a}$, Member
}

SUMMARY In orthogonal frequency-division multiplexing (OFDM)based wireless local area networks (WLANs), phase noise (PHN) and residual frequency offset (RFO) can cause the common phase error (CPE) and the inter-carrier interferences (ICI), which seriously degrade the performance of systems. In this letter, we propose a combined pilot symbol assisted and decision-directed channel estimation scheme based on the leastsquares (LS) and the maximum-likelihood (ML) algorithms. Simulation results present that the proposed scheme significantly improves the performance of OFDM-based WLANs.

key words: orthogonal frequency-division multiplexing (OFDM), channel estimation, phase noise (PHN), residual frequency offset (RFO), wireless local area networks (WLANs)

\section{Introduction}

Orthogonal frequency-division multiplexing (OFDM) has recently received considerable attention for its robustness against frequency selective channels. It has been adopted by the IEEE 802.11a standard as the transmission technique for high-rate wireless local area networks (WLANs) [1]. Moreover, the OFDM system is very sensitive to phase noise (PHN) [2]-[5] and residual frequency offset (RFO) due to imperfect synchronization [5], [6]. PHN and RFO cause both the common phase error (CPE) and the inter-carrier interferences (ICI), both of which impair the accurate channel estimation. Modeling PHN as a random process, two approaches are investigated in the literature. One can be obtained by measuring a real tuner with a phase-locked loop (PLL) [2]. The other does the typical PHN model of a freerunning oscillator [2]-[5]. We choose the latter model. In this letter, assuming that one packet is composed of one long training symbol and OFDM data symbols, we propose a channel estimation scheme based on the LS and the ML algorithms. Since it is hard to obtain the joint ML estimation of the CPE and the channel, we treat the CPE estimation and the channel estimation problems separately.

\section{System Model}

We consider an OFDM system with $N$ sub-carriers composed of a data sub-carrier set $S_{D}$ with $N_{D}$ sub-carriers, a pilot sub-carrier set $S_{P}$ with $N_{P}$ sub-carriers and a null sub-

\footnotetext{
Manuscript received October 6, 2005.

Manuscript revised December 8, 2005.

${ }^{\dagger}$ The authors are with Institute of New Media and Communications, School of Electrical Engineering and Computer Science, Seoul National University, Seoul, Korea.

a)E-mail: sckim@maxwell.snu.ac.kr

DOI: 10.1093/ietcom/e89-b.5.1709
}

carrier set $S_{N}$ with $N_{N}$ sub-carriers [1]. The time-domain OFDM symbol is generated by $N$-point IDFT and a cyclic prefix $(\mathrm{CP})$ of length $N_{C P}$ is appended at the beginning of each time-domain OFDM symbol.

Then, the received signal vector $\boldsymbol{r}_{m}$ at the $m$ th OFDM symbol over frequency selective fading channel, after $\mathrm{CP}$ removal, can be expressed as

$$
\boldsymbol{r}_{m}=\boldsymbol{F}_{\phi_{m}} \boldsymbol{F} \boldsymbol{X}_{m} \boldsymbol{H}_{m}+\boldsymbol{w}_{m}
$$

where $m=0,1, \cdots, N_{\text {packet }}-1$ is the OFDM symbol index and both PHN $\phi_{m}^{P H N}$ and RFO $\phi_{m}^{R F O}$ are represented by the matrix $\boldsymbol{F}_{\phi_{m}}$ given as

$$
\begin{aligned}
\boldsymbol{F}_{\phi_{m}}= & \operatorname{diag}\left\{e^{j\left(\phi_{m}^{P H N}(0)+\phi_{m}^{R F O}(0)\right)},\right. \\
& \left.\cdots, e^{j\left(\phi_{m}^{P H N}(N-1)+\phi_{m}^{R F O}(N-1)\right)}\right\} .
\end{aligned}
$$

$\phi_{m}^{P H N}$ in (2) can be modeled as a discrete Wiener process by $\phi_{m}^{P H N}(n)=\phi_{m-1}^{P H N}(N-1)+\sum_{t=-N_{C P}}^{n} u\left[m\left(N+N_{C P}\right)+t\right]$ where $\phi_{0}^{P H N}(n)=\sum_{t=-N_{C P}}^{n} u(t) . \quad u(t)$ in the expression for $\phi_{m}^{P H N}$ denotes a mutually independent Gaussian random variable with zero mean and variance $\sigma_{u}^{2}=2 \pi \beta T / N$ where $T$ is the OFDM symbol period and $\beta$ represents the phase noise linewidth [4]. $\phi_{m}^{R F O}$ in (2) can be modeled as an additional rotation due to the normalized RFO $v$ by $\phi_{m}^{R F O}(n)=\phi_{m-1}^{R F O}(N-1)+2 \pi v\left(N_{C P}+n\right) / N$ where $\phi_{0}^{R F O}(n)=2 \pi v\left(N_{C P}+n\right) / N$. In (1), $\boldsymbol{X}_{m}=\operatorname{diag}\left\{X_{m}(k)\right\}$ for $k \in S_{D} \cup S_{P}$ denotes a diagonal transmitted symbol matrix and $[\boldsymbol{F}]_{n, k}=\frac{1}{\sqrt{N}} \exp \left(j \frac{2 \pi n}{N} k\right)$ does the IDFT matrix where $k \in S_{D} \cup S_{P}$. Assuming ideal timing synchronization and sufficient $\mathrm{CP}$, the channel frequency response can be given as $\boldsymbol{H}_{m}=\boldsymbol{D} \boldsymbol{h}_{m}$ where $\boldsymbol{h}_{m}=\left[h_{m}(0) \cdots h_{m}(L-1)\right]^{T}$ denotes the channel impulse response (CIR) with $L$ multipaths and $[\boldsymbol{D}]_{k, l}=\exp \left(-j \frac{2 \pi k}{N} l\right)$ for $k \in S_{D} \cup S_{P}$ and $0 \leq l<L$. Moreover, the additive white Gaussian noise vector $\boldsymbol{w}_{m}=\left[w_{m}(0) \cdots w_{m}(N-1)\right]^{T}$ has the covariance matrix of $\sigma_{w}^{2} \boldsymbol{I}_{N}$ where $\boldsymbol{I}_{N}$ denotes an $N \times N$ identity matrix.

The frequency-domain received signal vector can be given as

$$
\boldsymbol{R}_{m}=\boldsymbol{F}^{H} \boldsymbol{r}_{m}=\varepsilon_{m} \boldsymbol{X}_{m} \boldsymbol{D} \boldsymbol{h}_{m}+\boldsymbol{n}_{I C I_{m}}+\boldsymbol{W}_{m}
$$

where (.) ${ }^{H}$ and $\boldsymbol{W}_{m}=\boldsymbol{F}^{H} \boldsymbol{w}_{m}$ denote the conjugated transpose and the frequency response of AWGN, respectively. It is seen that the CPE $\varepsilon_{m}$ in (3) affects all sub-carriers constantly, which is defined by

$$
\varepsilon_{m}=\frac{1}{N} \sum_{n=0}^{N-1} e^{j\left(\phi_{m}^{P H N}(n)+\phi_{m}^{R F O}(n)\right)} .
$$


The ICI vector $\boldsymbol{n}_{I C I_{m}}$ in (3) implies the ICI due to both PHN and RFO defined by $\boldsymbol{n}_{I C I_{m}}=\left(\boldsymbol{F}^{H} \boldsymbol{F}_{\phi_{m}} \boldsymbol{F}-\boldsymbol{\varepsilon}_{m} \boldsymbol{I}_{N_{D}+N_{P}}\right) \boldsymbol{X}_{m} \boldsymbol{D} \boldsymbol{h}_{m}$.

The autocorrelation of the CPE between adjacent OFDM symbols $\Phi_{\varepsilon}$ can be defined as

$$
\begin{aligned}
\Phi_{\varepsilon} & =E\left[\varepsilon_{m}^{*} \varepsilon_{m+1}\right] \\
& =\frac{1}{N^{2}} \sum_{p=0}^{N-1} \sum_{q=0}^{N-1} e^{j\left(\phi_{m+1}^{R F O}(q)-\phi_{m}^{R F O}(p)\right)} E\left[e^{j\left(\phi_{m+1}^{P H N}(q)-\phi_{m}^{P H N}(p)\right)}\right] .
\end{aligned}
$$

Since PHN is a discrete Wiener process, the difference $\phi_{m+1}^{P H N}(q)-\phi_{m}^{P H N}(p)$ in (5) is a zero-mean Gaussian random variable with variance equal to $\sigma_{u}^{2}\left|N+N_{C P}+q-p\right|$. After some algebraic manipulations, (5) can be given by

$$
\begin{aligned}
& \Phi_{\varepsilon}=\frac{e^{\left(j 2 \pi v / N-\sigma_{u}^{2} / 2\right)\left(N+N_{C P}\right)}}{N^{2}} \\
& \times\left\{2 \sum_{i=1}^{N} i \cosh \left((N-i)\left(j 2 \pi v / N-\sigma_{u}^{2} / 2\right)\right)-N\right\} .
\end{aligned}
$$

The $\varepsilon_{m}$ manifests mainly as a phase rotation $\theta_{\varepsilon_{m}}=\angle\left(\varepsilon_{m}\right)$ for slow PHN and RFO processes [5]. Therefore, it can be expressed as $\varepsilon_{m}=\varepsilon_{m}^{(\text {res })} \varepsilon_{m-1}$ where $\varepsilon_{m}^{(\text {res })}$ is the multiplication factor to imply a residual CPE term, which defines the relation between adjacent OFDM symbols.

The transmitted symbol $X_{m}(k)$ is assumed as a random variable with zero mean and variance $\sigma_{x}^{2}=E\left[|X(k)|^{2}\right]$. Furthermore, without any loss of generality, the channel frequency response can be assumed to satisfy $E\left[\left|H_{m}(k)\right|^{2}\right]=$ 1. The ICI vector $\boldsymbol{n}_{I C I_{m}}$ in (3) can be approximated as a zero-mean Gaussian vector of the covariance matrix of $\sigma_{I C I}^{2} \boldsymbol{I}_{N_{D}+N_{P}}$ [3], [6]. Here, we define the ICI-plus-noise vector as $\boldsymbol{V}_{m}=\boldsymbol{n}_{I C I_{m}}+\boldsymbol{W}_{m}$ and assume that $\boldsymbol{V}_{m}$ is a zero mean Gaussian vector with the covariance matrix of $\sigma_{V}^{2} \boldsymbol{I}_{N_{D}+N_{P}}=$ $\left(\sigma_{I C I}^{2}+\sigma_{w}^{2}\right) \boldsymbol{I}_{N_{D}+N_{P}}$.

\section{Channel Estimation}

By defining a new effective CIR vector $\boldsymbol{h}_{m}^{\text {eff }}=\varepsilon_{m} \boldsymbol{h}_{m}$, (3) is rewritten by

$$
\boldsymbol{R}_{m}=\boldsymbol{X}_{m} \boldsymbol{D} \boldsymbol{h}_{m}^{e f f}+\boldsymbol{V}_{m}=\boldsymbol{X}_{m} \boldsymbol{H}_{m}^{e f f}+\boldsymbol{V}_{m}
$$

where $\boldsymbol{H}_{m}^{\text {eff }}=\boldsymbol{D} \boldsymbol{h}_{m}^{\text {eff }}$ is the effective channel frequency response vector. When the variation of the CIR between adjacent OFDM symbols is negligible compared with $\Phi_{\varepsilon}$, the effective channel frequency response can be approximated as

$$
\boldsymbol{H}_{m}^{e f f} \approx \varepsilon_{m}^{(r e s)} \boldsymbol{H}_{m-1}^{e f f} .
$$

Under the assumption that the effective CIR vector $\boldsymbol{h}_{m}^{\text {eff }}$ is a deterministic but unknown vector, the proposed channel estimation scheme is implemented by the following steps. Step 1) Exploiting a BPSK modulated long training symbol $\boldsymbol{X}_{0}$, estimate the effective CIR $\hat{\boldsymbol{h}}_{0}^{\text {eff }}$ by [7]

$$
\begin{aligned}
\hat{\boldsymbol{h}}_{0}^{e f f} & =\left(\boldsymbol{D}^{H} \boldsymbol{D}\right)^{-1} \boldsymbol{D}^{H} \boldsymbol{X}_{0}^{-1} \boldsymbol{R}_{0} \\
& =\boldsymbol{h}_{0}^{e f f}+\left(\boldsymbol{D}^{H} \boldsymbol{D}\right)^{-1} \boldsymbol{D}^{H} \boldsymbol{X}_{0}^{-1} \boldsymbol{V}_{0}=\boldsymbol{h}_{0}^{e f f}+\boldsymbol{V}_{0}^{\prime}
\end{aligned}
$$

where $\boldsymbol{V}_{0}^{\prime}=\left(\boldsymbol{D}^{H} \boldsymbol{D}\right)^{-1} \boldsymbol{D}^{H} \boldsymbol{X}_{0}^{-1} \boldsymbol{V}_{0}$ is a zero-mean Gaussian noise with the covariance matrix of $\boldsymbol{C}_{V^{\prime}}=\sigma_{V}^{2}\left(\left(\boldsymbol{D}^{H} \boldsymbol{D}\right)^{-1}\right)^{H}$. Note that the CIR length $L$ should be known in order to construct $\left(\boldsymbol{D}^{H} \boldsymbol{D}\right)^{-1} \boldsymbol{D}^{H}$ in (9). Here, we simply assume that the estimate of $L$ is equal to the CIR length [8]. Then, $\hat{\boldsymbol{H}}_{0}^{\text {eff }}=\boldsymbol{D} \hat{\boldsymbol{h}}_{0}^{e f f}$. Increase $m$ by 1 .

Step 2) Estimate the phase of $\varepsilon_{m}^{\text {(res) }}$ using (8) by the LS method as $\hat{\theta}_{\varepsilon_{m}^{(r e s)}}=\angle\left(\left(\boldsymbol{X}_{m, P} \hat{\boldsymbol{H}}_{m-1, P}^{\text {eff }}\right)^{H} \boldsymbol{R}_{m, P}\right)$ where $\boldsymbol{R}_{m, P}, \boldsymbol{X}_{m, P}$ and $\hat{\boldsymbol{H}}_{m-1, P}^{\text {eff }}$ denote the received signal vector at the pilot sub-carriers, the diagonal pilot symbol matrix and the effective channel frequency response estimate vector composed of only pilot sub-carriers, respectively. Then, $\hat{\boldsymbol{H}}_{m}^{\text {eff }}=$ $\exp \left(j \hat{\theta}_{\left.\varepsilon_{\text {ress }}\right)}\right) \hat{\boldsymbol{H}}_{m-1}^{\text {eff }}$.

Step 3) Demodulate the OFDM symbol using the current $\hat{\boldsymbol{H}}_{m}^{\text {eff }}$ and make the tentative symbol matrix $\tilde{\boldsymbol{X}}_{m}$.

Step 4) Given $\tilde{\boldsymbol{X}}_{m}$, which is assumed to be the same as the transmitted symbol matrix $\boldsymbol{X}_{m}$, the normalized received vector $\overline{\boldsymbol{R}}_{m}$ from (7) can be defined by

$$
\overline{\boldsymbol{R}}_{m}=\left(\tilde{\boldsymbol{X}}_{m}\right)^{-1} \boldsymbol{R}_{m}=\boldsymbol{D} \boldsymbol{h}_{m}^{\text {eff }}+\left(\tilde{\boldsymbol{X}}_{m}\right)^{-1} \boldsymbol{V}_{m} .
$$

The normalized received vector $\overline{\boldsymbol{R}}_{m}$ is Gaussian with mean $\boldsymbol{D} \boldsymbol{h}_{m}^{\text {eff }}$ and covariance matrix $\boldsymbol{C}_{\bar{R}_{m}}=\sigma_{V}^{2} E\left[\left|\boldsymbol{X}_{m}^{-1}\right|^{2}\right]$. Thus, the ML based effective CIR estimate is given by [7]

$$
\begin{aligned}
\hat{\boldsymbol{h}}_{m}^{e f f} & =\left(\boldsymbol{D}^{H} \boldsymbol{C}_{\bar{R}_{m}}^{-1} \boldsymbol{D}\right)^{-1} \boldsymbol{D}^{H} \boldsymbol{C}_{\bar{R}_{m}^{-1}} \overline{\boldsymbol{R}}_{m} \\
& =\left(\boldsymbol{D}^{H} \boldsymbol{C}_{X^{-1}}^{-1} \boldsymbol{D}\right)^{-1} \boldsymbol{D}^{H} \boldsymbol{C}_{X^{-1}}^{-1} \overline{\boldsymbol{R}}_{m} .
\end{aligned}
$$

where $\boldsymbol{C}_{X^{-1}}=E\left[\left|\boldsymbol{X}_{m}^{-1}\right|^{2}\right]$ is the diagonal matrix consists of $E\left[\left|1 / X_{m}(k)\right|^{2}\right]$ for $k \in S_{D}$ and 1.0 for $k \in S_{P}$. When the average symbol energy $\sigma_{x}^{2}$ is normalized to unity, $E\left[|1 / X(k)|^{2}\right]$ can be shown to have values of 1.0 for PSK signaling, 1.8889 for 16-QAM signaling and 2.6854 for 64-QAM signaling. Then, $\hat{\boldsymbol{H}}_{m}^{\text {eff }}=\boldsymbol{D} \hat{\boldsymbol{h}}_{m}^{\text {eff }}$.

Step 5) Re-estimate the transmitted symbol matrix $\hat{\boldsymbol{X}}_{m}$ using the current $\hat{\boldsymbol{H}}_{m}^{\text {eff }}$.

Step 6) Go to Step 2) with $m \leftarrow m+1$.

Assuming that $\left(\boldsymbol{D}^{H} \boldsymbol{C}_{X^{-1}}^{-1} \boldsymbol{D}\right)^{-1} \boldsymbol{D}^{H} \boldsymbol{C}_{X^{-1}}^{-1}$ in (11) is precomputed, complexity reduction can be obtained by using FFT pruning or transform decomposition [9].

\section{Simulation Results}

In this section, we present simulation results in the uncoded OFDM system with PHN and RFO. Part of simulation parameters is based on the IEEE 802.11a standard [1], which is summarized as follows.

- The DFT size $N$ is 64 , the number of data sub-carriers $N_{D}$ is 48 , the number of pilot sub-carriers $N_{P}$ is 4 , and the number of null sub-carriers $N_{N}$ is 12 .

- The CP length $N_{C P}$ is 16 . 


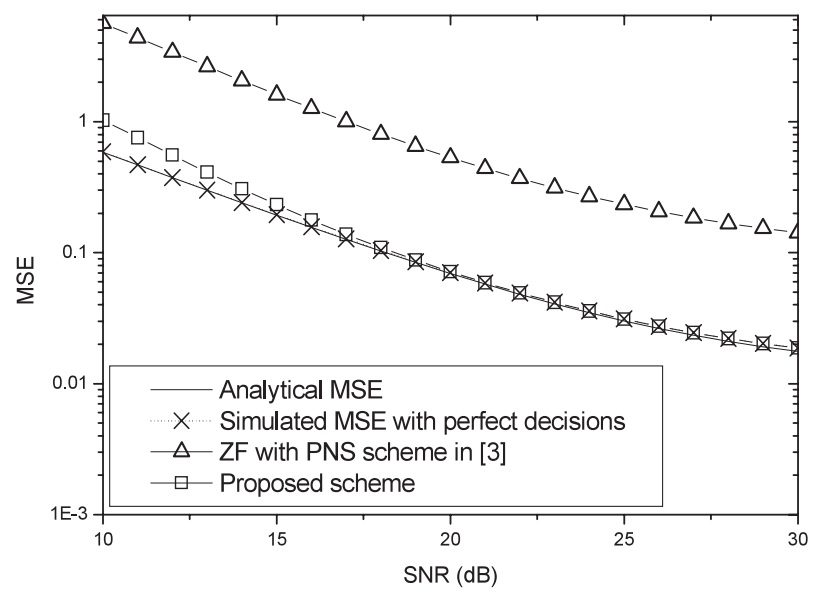

Fig. 1 MSE performance of the effective channel frequency response estimators at $N_{\text {packet }}=21$ in static channels.

- Each BPSK modulated pilot sub-carrier is located at $k=-21,-7,7$ and 21 .

- The OFDM data sub-carriers are modulated using 16QAM.

- The OFDM symbol period $T$ is $4.0 \mu \mathrm{s}$.

We consider frequency-selective Rayleigh fading channels with exponential power delay profile given by $E\left[|h(l)|^{2}\right]=e^{-l / 4} / \sum_{l=0}^{L-1} e^{-l / 4}$ where $0 \leq l<L$ and $L=4$. We use PHN with $2 \pi \beta T=0.01$ and the normalized RFO $v=0.01$. In the simulation, the average signal-to-noise ratio (SNR) is defined as $52 \sigma_{x}^{2} / 64 \sigma_{w}^{2}$. The ZF represents the zero-forcing equalization (one-tap equalization) exploiting the long training symbol in the packet preamble [6]. In this case, the channel can be estimated as $\hat{\boldsymbol{H}}=\boldsymbol{X}^{-1} \boldsymbol{R}$.

At first, we consider static channels which indicate that the CIR is constant over a packet but varies independently from one packet to the next. Regarding the accuracy of the effective channel frequency response estimator, we define the mean square error (MSE) at the OFDM symbol as $M S E=E\left[\left|\hat{\boldsymbol{H}}_{m}^{e f f}-\boldsymbol{H}_{m}^{e f f}\right|^{2}\right]$. Figure 1 shows the MSE performance of the effective channel frequency response estimators. The MSE with perfect tentative symbol matrix $\tilde{\boldsymbol{X}}_{m}$ can be analytically calculated as $\sigma_{V}^{2} \operatorname{Tr}\left\{\boldsymbol{D}\left(\left(\boldsymbol{D}^{H} \boldsymbol{C}_{X^{-1}}^{-1} \boldsymbol{D}\right)^{-1}\right)^{H} \boldsymbol{D}^{H}\right\}$ where $\operatorname{Tr}\{\cdot\}$ indicates the trace of a matrix. It is seen that the proposed scheme outperforms the ZF with the phase noise suppression (PNS) scheme [3] in all ranges of SNR. In Fig. 1, the MSE performance of the proposed scheme is close to the simulated MSE with perfect tentative decisions. More explicitly, at low SNR values the achievable performance is degraded by the associated decision-error propagation, however tentative decisions become reliable for SNRs in excess of $20 \mathrm{~dB}$. In Fig. 2, we present the SER performance versus SNR. The performance of the proposed scheme is also compared with the ideal performance without PHN and RFO, the performance with the ideal effective CIR, the performance of the PNS scheme with the ideal CIR, and that of the ZF with the PNS scheme. It can be concluded that it achieves almost the performance of the PNS scheme

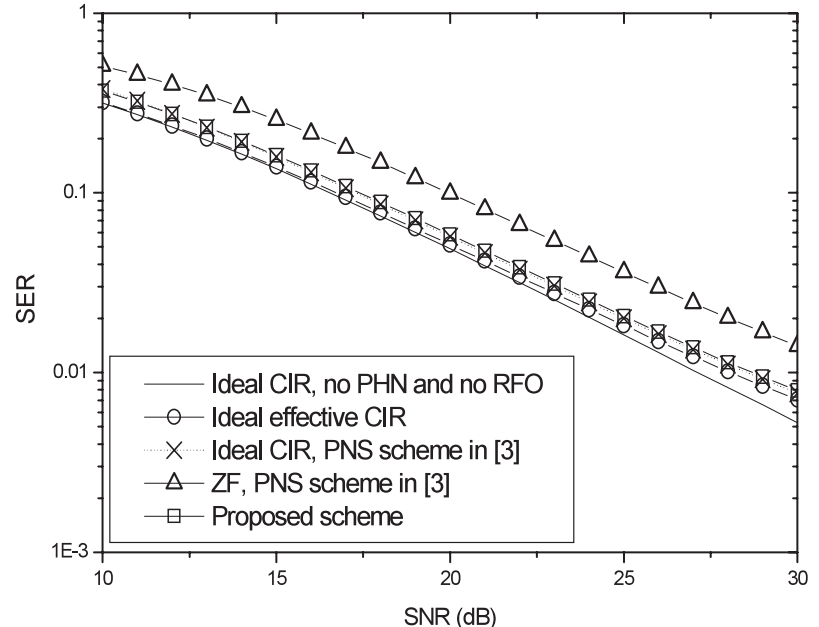

Fig. 2 SER performance versus SNR at $N_{\text {packet }}=21$ in static channels.

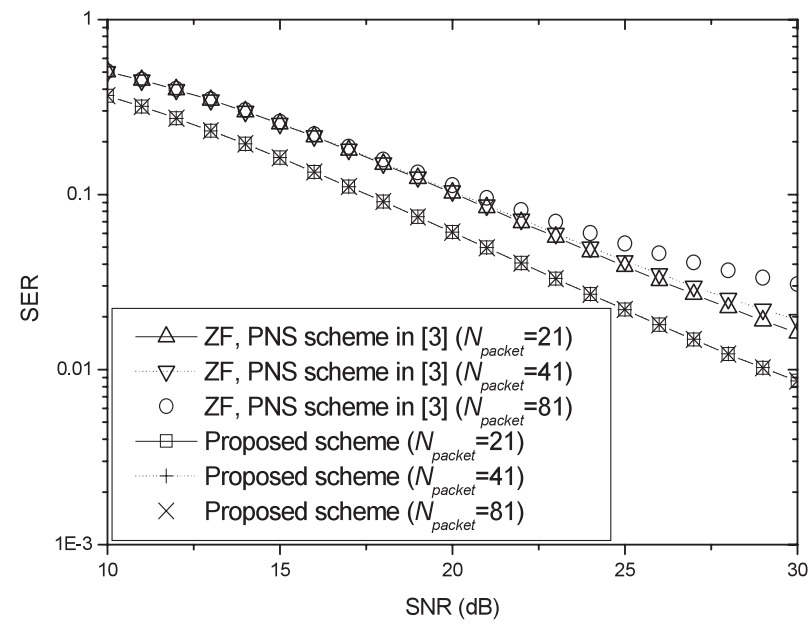

Fig. 3 SER performance with the packet size of $N_{\text {packet }}$ in slowly time-varying channels.

with the ideal CIR and is found to prevent remarkably the performance degradation due to PHN, RFO and channel estimation errors.

Figure 3 shows the SER degradation due to the packet length of $N_{\text {packet }}$ in slowly time-varying channels. In our simulation, it is assumed that slowly time-varying channels follow the Jakes' model [10], the carrier frequency is set to be $5.8 \mathrm{GHz}$, and the user terminal is moving at speed $3 \mathrm{~m} / \mathrm{s}$. Since the ZF with the PNS scheme uses the channel information estimated at the head of the packet, when the packet length of $N_{\text {packet }}$ increases, the SER degradation of the ZF with the PNS scheme increases due to channel estimation errors. In the proposed scheme, the residual CPE estimate is obtained using the pilot symbols in each OFDM symbol and the effective CIR is estimated in the decision-directed manner. Therefore, both the time-varying $\mathrm{CPE}$ and a timevarying channel can be tracked using the proposed scheme and the proposed scheme over slowly time-varying channels is robust with the increase of the packet length $N_{\text {packet }}$. 


\section{Conclusion}

In this letter, we proposed a channel estimation scheme for OFDM-based WLANs. The proposed scheme significantly compensates the performance degradation due to channel estimation errors, PHN and RFO. Moreover, the proposed scheme is robust in static or slowly time-varying channels compared with other scheme.

\section{Acknowledgments}

This work was supported partly by Brain Korea 21 Project in 2005 and partly by ITRC.

\section{References}

[1] IEEE 802.11 WG, part 11: Wireless LAN Medium Access Control (MAC) and Physical Layer (PHY) Specifications: High-speed Physical Layer in the $5 \mathrm{GHz}$ band, Supplement to IEEE 802.11 Standard, Sept. 1999.

[2] L. Piazzo and P. Mandarini, "Analysis of phase noise effects in
OFDM modems," IEEE Trans. Commun., vol.50, no.10, pp.16961705, Oct. 2002.

[3] S. Wu and Y. Bar-Ness, "A phase noise suppression algorithm for OFDM-based WLANs," IEEE Commun. Lett., vol.6, no.12, pp.535-537, Dec. 2002.

[4] S. Wu and Y. Bar-Ness, "OFDM systems in the presence of phase noise: Consequences and solutions," IEEE Trans. Commun., vol.52, no.11, pp.1988-1996, Nov. 2004.

[5] K. Nikitopoulos and A. Polydoros, "Phase-impairment effects and compensation algorithms for OFDM systems," IEEE Trans. Commun., vol.53, no.4, pp.698-707, April 2005.

[6] H. Cheon and D. Hong, "Effect of channel estimation error in OFDM-based WLAN," IEEE Commun. Lett., vol.6, no.5, pp.190192, May 2002.

[7] S.M. Kay, Fundamentals of statistical signal processing: Estimation theory, Prentice-Hall, Englewood Cliffs, NJ, 1993.

[8] R. van Nee and R. Prasad, OFDM for Wireless Multimedia Communications, Artech House Publisher, 2000.

[9] H.V. Sorensen and C.S. Burrus, "Efficient computation of the DFT with only a subset of input or output points," IEEE Trans. Signal Process., vol.41, pp.1184-1200, March 1993.

[10] W.C. Jakes, Microwave mobile communications, Wiley, New York, 1974. 